\title{
Germline mutation analyses of malignant ground glass opacity nodules in non-smoking lung adenocarcinoma patients
}

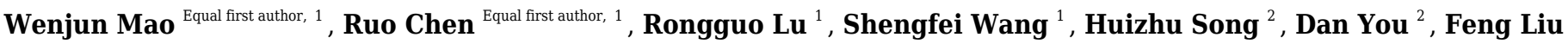 \\ ${ }^{1}$, Yijun He ${ }^{1}$, Mingfeng Zheng ${ }^{\text {Corresp. } 1}$ \\ ${ }^{1}$ Department of Cardiothoracic Surgery, The Affiliated Wuxi People's Hospital of Nanjing Medical University, Wuxi, Jiangsu, China \\ 2 Department of Pharmacy, The Affiliated Wuxi People's Hospital of Nanjing Medical University, Wuxi, Jiangsu, China \\ Corresponding Author: Mingfeng Zheng \\ Email address: zhengmfmedical@126.com
}

Background. Germline mutations play an important role in the pathogenesis of lung cancer. Nonetheless, research on malignant ground glass opacity (GGO) nodules is limited.

Methods. A total of 13 participants with malignant GGO nodules were recruited in this study. Peripheral blood was used for exome sequencing, and germline mutations were analyzed using InterVar. The whole exome sequencing dataset was analyzed using a filtering strategy. KOBAS 3.0 was used to analyze KEGG pathway to further identify possible deleterious mutations. Results. There were 7 potentially deleterious germline mutations. NM_001184790:exon8: c.C1070T in PARD3, NM_001170721:exon4:c.C392T in BCAR1 and NM_001127221:exon46: C.G6587A in CACNA1A were present in three cases each; rs756875895 frameshift in MAX, NM_005732: exon13:c.2165_2166insT in RAD50 and NM_001142316:exon2:c.G203C in LMO2, were present in two cases each; one variant was present in NOTCH3. Conclusions. Our results expand the germline mutation spectrum in malignant GGO nodules. Importantly, these findings will potentially help screen the highrisk population, guide their health management, and contribute to their clinical treatment and determination of prognosis. 


\section{Germline mutation analyses of malignant ground glass opacity nodules} 2 in non-smoking lung adenocarcinoma patients

3

4

5 6

7

8 9 10

11

12

13 14

15 16

17

18

19

20
Wenjun Mao ${ }^{1 \uparrow}$, Ruo Chen ${ }^{1 \uparrow, ~ R o n g g u o ~ L u ¹, ~ S h e n g f e i ~ W a n g ~}{ }^{1}$, Huizhu Song ${ }^{2}$, Dan You ${ }^{2}$, Feng Liu ${ }^{1}$, Yijun $\mathrm{He}^{1}$, Mingfeng Zheng ${ }^{1 *}$

${ }^{1}$ Department of Cardiothoracic Surgery, The Affiliated Wuxi People's Hospital of Nanjing Medical University, Wuxi, Jiangsu, China

${ }^{2}$ Department of Pharmacy, The Affiliated Wuxi People's Hospital of Nanjing Medical University, Wuxi, Jiangsu, China

I The authors contributed equally to this work.

Corresponding Author:

Mingfeng Zheng

No. 299 Qing Yang Road, Wuxi, Jiangsu, 214023, China

Email address: zhengmfmedical@126.com 


\section{Abstract}

22 Background. Germline mutations play an important role in the pathogenesis of lung cancer.

23 Nonetheless, research on malignant ground glass opacity (GGO) nodules is limited.

24 Methods. A total of 13 participants with malignant GGO nodules were recruited in this study.

25 Peripheral blood was used for exome sequencing, and germline mutations were analyzed using 26 InterVar. The whole exome sequencing dataset was analyzed using a filtering strategy. KOBAS 273.0 was used to analyze KEGG pathway to further identify possible deleterious mutations.

28 Results. There were 7 potentially deleterious germline mutations. NM_001184790:exon8:

29 c.C1070T in PARD3, NM_001170721:exon4:c.C392T in BCAR1 and NM_001127221:exon46:

30 c.G6587A in $C A C N A 1 A$ were present in three cases each; rs756875895 frameshift in $M A X$,

31 NM_005732: exon13:c.2165_2166insT in RAD50 and NM_001142316:exon2:c.G203C in

32 LMO2, were present in two cases each; one variant was present in NOTCH3.

33 Conclusions. Our results expand the germline mutation spectrum in malignant GGO nodules.

34 Importantly, these findings will potentially help screen the high-risk population, guide their

35 health management, and contribute to their clinical treatment and determination of prognosis. 
82

\section{Introduction}

Though therapeutic advances have been made using targeted therapy and immunotherapy, lung cancer continues to be the most common cause of cancer-related deaths worldwide (Siegel, Miller, \& Jemal, 2015). The majority of lung cancers are caused by somatic mutations that accumulate with age and germline mutations could explain a predisposition to cancer development.

Lung cancer is a complex disease that is mainly attributed to smoking (Hung et al., 2008). However, over $10 \%$ of lung cancer patients are non-smokers (Subramanian \& Govindan, 2007). The development of lung cancer in never-smokers is associated with several potential risk factors, including environmental pollution and genetic predisposition (Malhotra, Malvezzi, Negri, La Vecchia, \& Boffetta, 2016). Germline mutations in lung cancer have been studied to some extent (Ikeda et al., 2014; Liu et al., 2016; Shukuya et al., 2018; Zhang et al., 2017), - including some in familial settings (Kanwal et al., 2018; Tomoshige et al., 2015). There are also studies on non-smoking lung cancer cohorts (Donner et al., 2018; Renieri et al., 2014). Nonetheless, studies on germline mutations in lung cancer patients fall far short when compared to those on somatic mutations. There is a need to study germline mutations in lung cancer since they are related to the pharmacodynamics, prognosis, and interactions with somatic mutations (Bartsch, Dally, Popanda, Risch, \& Schmezer, 2007; Erdem, Giovannetti, Leon, Honeywell, \& Peters, 2012; Wang et al., 2018; Winther-Larsen et al., 2015).

GGOs observed on computed tomography are described as hazy areas but preserved broncho-vascular markings (Austin et al., 1996; Lee et al., 2014). Advances in high resolution computed tomography and its application in lung cancer screening have led to an increased detection rate of GGOs, with an estimated prevalence of $0.2-0.5 \%$ (Henschke et al., 2006). While many GGOs are benign and disappear with time, some are persistent and turn malignant. These tumours are frequently found in non-smokers and women lung cancer patients (Blons et al., 2006; Raz, He, Rosell, \& Jablons, 2006).

Here, we recruited a total of 13 non-smoking patients with malignant GGO nodules to study their germline mutations using whole exome sequencing (WES). The results provide a better understanding of molecular mechanisms underlying the development of GGOs and their predisposition to turn cancerous.

\section{Materials \& Methods}

\section{Study subjects}

Candidates that were radiologically found to have small GGO nodules in physical checkup or who came to outpatients department for the reason of cough and checked by computed tomography to have small GGO nodules were closely followed up from 6 months to 3 years. When the GGO nodules increased in size or the nodule density increased or the solid components of pulmonary nodules increased, 13 patients were recruited and underwent surgery and thereafter they were histologically confirmed to have malignant GGOs in the Department of Cardiothoracic Surgery at Wuxi People's Hospital affiliated to Nanjing Medical University, China, from April 1 ${ }^{\text {st }}, 2019$ to August 30 th, 2019 . No other treatments were adopted. Written informed consent was obtained from all participants. Blood samples were collected before surgery and their clinical information was recorded. The research project was approved by the institutional review board of Wuxi People's Hospital affiliated to Nanjing Medical University (no: HS2019014).

\section{DNA extraction, library preparation, capture enrichment, and WES}


84

85

86

87

88

89

90

91

92

93

94

95

96

97

98

99

100

101

102

103

104

105

106

107

108

109

110

111

112

113

114

115

116

117

118

119

120

121

122

123

124

125

126

127

128

129

Genomic DNA was extracted from peripheral blood collected from participants using a DNA blood mini kit (Qiagen, Germantown, MD, USA) following the manufacturer's instructions. DNA concentration and purity were assessed by a Qubit fluorometer (Invitrogen, Carlsbad, CA, USA).

WES was conducted on $500 \mathrm{ng}$ of genomic DNA from each participant. Fragment libraries were prepared from sheared samples by sonication, and exons were enriched by hybridisation capture with a SureSelect Human All Exon V6 Kit (Agilent, Santa Clara, CA, USA) according to the manufacturer's protocol. Captured DNA was amplified followed by solid-phase bridge amplification. The paired-end library was sequenced on a NovaSeq 6000 platform (Illumina, San Diego, CA, USA). The data from this study were deposited in NCBI Sequence Read Archive under SRA accession: PRJNA613408 (https://www.ncbi.nlm.nih.gov/bioproject/PRJNA613408).

\section{Read alignment, variant calling, variant annotation, and filtering}

Trimmomatic-0.36 (Bolger, Lohse, \& Usadel, 2014) was used as quality control for raw data and to remove adapters. Clean sequence reads were aligned to the human reference genome (GRCh37/b37 assembly) using Burrows-Wheeler Aligner software (version 0.7.10) (Li \& Durbin, 2009). Picard (version 2.9.2, Broad Institute, Boston, MA, USA) was used to remove duplicates. Variant detection was performed using HaplotypeCaller in the Genome Analysis Toolkit 3.4 (www.broad institute.org/gatk) (DePristo et al., 2011). Variants were annotated using InterVar database. Detailed stepwise filtering strategy for screening potential candidate germline mutations was described in supplement 1.

\section{KEGG pathway analysis}

KEGG pathway analysis was conducted via KOBAS 3.0 (http://kobas.cbi.pku.edu.cn/kobas3/genelist/)

\section{Results}

Clinical information of patients was summarised in Table 1. The mean age at onset of nonsmall cell lung cancer (NSCLC) in the cases was 61.5 years (range $48-79$ years). All cases were non-smokers and $84.6 \%$ were females.

Two computed tomographic images are shown as representative of GGO nodules in the study cohort (Fig 1). Of the 13 cases, 12 were diagnosed as lung adenocarcinomas while one was diagnosed as an atypical adenomatous hyperplasia. Five cases were adenocarcinoma in situ, four were invasive, and three were minimally invasive. Eight of the GGO nodules were located at the right upper lobe, two were at the right lower lobe, and three were at the left upper lobe. Detailed histologic information is presented in Table 2.

We used a stepwise filtering strategy to screen for potential candidate variants (Fig 2). Of 83,302 single-nucleotide variants (SNVs) located in exons of the whole exome, our filtering strategy identified 17 potential candidate variants (Table 3). Of the 17 candidate variants in 17 genes, NM_000700:exon6:c.A418T in AXAN1, NM_001184790:exon8:c.C1070T in PARD3, NM_001170721:exon4:c.C392T in BCAR̄, NM_001127221:exon46:c.G6587A in CACNA1A, NM_001170634:exon5:c.G383A in FUS, and NM_002451:exon6:c.C538T in $M T A P$ were present in three cases each. In addition, rs756875895 frameshift in MAX, NM_001199292:exon7:c.C482G in HSD17B4, NM_005732:exon13:c.2165_2166insT in RAD50, NM_001350128:exon11:c.T1172C in PPOX, NM_001098816:exon28:c.A4751G in TENM4, NM_004004:exon2:c.235delC in GJB2, and NM_001142316:exon2:c.G203C in LMO2 were present in two cases each. The remaining variants were present in one case each. Of the 17 variants, 12 were nonsynonymous mutations, four were frameshift deletions, and one was a stopgain. The distribution of mutations in each patient was shown in Fig 3. 
130

131

132

133

134

135

136

137

138

139

140

141

142

143

144

145

146

147

148

149

150

151

152

153

154

155

156

157

158

159

160

161

162

163

164

165

166

167

168

169

170

171

172

173

174

We identified four potential deleterious frameshift deletions: rs80338943 in GJB2, rs587781454 in RAD50, rs756875895 in $M A X$, each occurring in two cases, and a frameshift in LDLRAP1, occurring in one case. The first frameshift, rs 80338943 in GJB2, causing a p.L79fs fusion, was annotated as uncertain significance by InterVar. The second frameshift, rs 587781454 in RAD50 caused a p.K722fs fusion and was annotated as pathogenic from InterVar. The third frameshift, rs756875895 in $M A X$, was annotated as likely pathogenic in InterVar, causing a fusion of $\mathrm{p} . \mathrm{L} 52 \mathrm{fs}$. The frameshift in LDLRAP1 caused a fusion of $\mathrm{p}$.W22fs in one case and was interpreted as pathogenic from InterVar, it might be deleterious in the development of lung cancer. Another potential deleterious variant was a stopgain, rs7755898 in CYP21A2, causing a protein change of p.Q289X which was likely pathogenic according to InterVar.

The other interesting candidates were four likely pathogenic SNVs annotated from InterVar: NOTCH 3:p.T357M (present in one case), HSD17B4 p.A161G (present in two cases), PPOX p.L391P (present in two cases), and TENM4 p.Q1584R (present in two cases).

There were five SNVs annotated as uncertain significance by InterVar that were present in three patients: ANXA1:p.I140 F, BCAR1:p.P131L, CACNA1A:p.R2196Q, FUS:p.S128N, and MTAP:p.R180W.

There were two additional candidate variants, LMO2 p.G68A and TTN p.R18629C, that were present in two cases and one case, respectively (Table 3). Their annotations by InterVar were of uncertain significance.

KEGG analysis did not indicate pathways that were related to $A X A N 1, T E N M 4$ and GJB2. Pathways of BCAR1, CYP21A2, LPLRAP1, HSD17B4, MTAP, PPOX and TTN were not associated with cancer. Pathways derived from NOTCH3, PARD3, CACNA1A, MAX, RAD50, FUS and $L M O 2$ were cancer-related. The details were shown in a supplemental table (Table S). Mutations in these genes were considered unlikely to cause cancer, therefore they would not be discussed here.

\section{Discussion}

Although there are studies available on genetic mutations of lung cancer, the heritability of lung cancer, especially for GGO nodules, remains understudied compared to sporadic lung cancer. Using WES, our study reports germline mutations in GGO nodules of non-smoker lung cancer patients, largely females.

The discovery of germline mutations is very significant for both basic research and clinical treatment of lung cancer. First, germline mutations may play a role in tumorigenesis. Wang et al. (Wang et al., 2018) reported that germline mutations interacted with somatic mutations, indicating their role in lung tumorigenesis. Tomoshige et al. (Tomoshige et al., 2015) also reported that germline mutations could cause familial lung cancer. Second, germline mutations are valuable for prognosis (Erdem et al., 2012). For example, a study by Winther-Larsen et al. (Winther-Larsen et al., 2015) found that genetic polymorphism in the epidermal growth factor receptor could predict the outcome in advanced NSCLC patients treated with erlotinib. Third, germline mutations are closely associated with a genetic predisposition to cancer, and screening for germline mutations is beneficial to the susceptible population (Chen et al., 2015) and for their health management.

In this study, we used a highly selective population, lung adenocarcinoma patients with GGOs, to investigate germline mutations and their possible role in the predisposition to lung cancer. In our cohort, 11 of 13 were females and all were non-smokers. The ethnicity of all patients was Han Chinese. The aforementioned facts were consolidated with the notion that 
175

176

177

178

179

180

181

182

183

184

185

186

187

188

189

190

191

192

193

194

195

196

197

198

199

200

201

202

203

204

205

206

207

208

209

210

211

212

213

214

215

216

217

218

219

220

221

malignant GGO nodules occur frequently in non-smokers and women (Blons et al., 2006; Raz et al., 2006).

Strong evidence for two deleterious germline mutations (rs587781454 in RAD50 and rs 756875895 in $M A X$ ) has been shown in lung cancer patients. rs587781454 in RAD50 was reported as a hereditary predisposition and labelled as pathogenic in ClinVar (Nykamp et al., 2017). rs 756875895 in MAX was labelled as likely pathogenic by InterVar. Both variants occurred simultaneously in two females (WL-5 and WL-6). Both had minimally invasive GGO nodules. How these mutations in the same patient affected lung tumorigenesis is worth examining.

There was one likely pathogenic variant in NOTCH 3 (WL-13). The expression of NOTCH 3 was inversely associated with the sensitivity to platinum-based chemotherapy in patients with NSCLC. The NOTCH 3 protein, rather than the gene polymorphism, is associated with the chemotherapy response and prognosis of advanced NSCLC patients (Shi, Qian, Ma, Zhang, \& Han, 2014).

Though annotated as uncertain significance by InterVar, three patients carried variants in $B C A R 1$ (WL-7, WL-10 and WL-13) and CACNA1A (WL-5, WL-6 and WL-9). Increased expression of $B C A R 1$ was associated with poor prognosis and carcinogenesis in NSCLC (Deng, Sun, Jason, \& Yang, 2013; Huang et al., 2012). Overexpression of CACNA1A predicted a poor prognosis in NSCLC (Zhou et al., 2017). There were one additional candidate variants, LMO2 p.G68A in WL-1 and WL-8. Collectively, these findings suggest that germline mutations may function by regulating gene expression and thereby affect cancer development and/or prognosis. Our study has limitations. First, the sample size is small. In our study, only non-smoker patients with malignant GGOs were enrolled. Second, gene expression was not investigated. Finally, the identified germline mutations have not been validated. These limitations restrict conclusions about their causative effects on tumorigenesis and their roles as biomarkers for prognosis or for treatment response.

\section{Conclusions}

In summary, our results demonstrate potentially deleterious germline mutations in GGO nodules in non-smoking lung adenocarcinoma patients. These findings significantly expand the spectrum of genetic variants that may affect the response to therapies and patient survival and possibly increase the risk of being germline mutation carriers. However, due to the small patient samples, our observations encourage further studies. In future, prospective studies, expanding enrolled patients and functional studies should be performed to better understand their causative roles in tumorigenesis and prognosis, and to better manage patients' health.

\section{Acknowledgements}

We thank Editage (www.editage.cn) for the language editing.

\section{References}

Austin, J. H., Muller, N. L., Friedman, P. J., Hansell, D. M., Naidich, D. P., Remy-Jardin, M., . . Zerhouni, E. A. (1996). Glossary of terms for CT of the lungs: recommendations of the Nomenclature Committee of the Fleischner Society. Radiology, 200(2), 327-331. doi:10.1148/radiology.200.2.8685321

Bartsch, H., Dally, H., Popanda, O., Risch, A., \& Schmezer, P. (2007). Genetic risk profiles for cancer susceptibility and therapy response. Recent Results Cancer Res, 174, 19-36. doi:10.1007/978-3-540-37696-5_2

Blons, H., Cote, J. F., Le Corre, D., Riquet, M., Fabre-Guilevin, E., Laurent-Puig, P., \& Danel, C. (2006). Epidermal growth factor receptor mutation in lung cancer are linked to bronchioloalveolar differentiation. Am J Surg Pathol, 30(10), 1309-1315. doi:10.1097/01.pas.0000213285.65907.31

Bolger, A. M., Lohse, M., \& Usadel, B. (2014). Trimmomatic: a flexible trimmer for Illumina sequence data. Bioinformatics, 30(15), 2114-2120. doi:10.1093/bioinformatics/btu170

PeerJ reviewing PDF | (2021:01:57035:2:0:NEW 8 Jul 2021) 
222

223

224

225

226

227

228

229

230

231

232

233

234

235

236

237

238

239

240

241

242

243

244

245

246

247

248

249

250

251

252

253

254

255

256

257

258

259

260

261

262

263

264

265

266

267

268

269

270

271

272

273

274

Chen, H. Y., Yu, S. L., Ho, B. C., Su, K. Y., Hsu, Y. C., Chang, C. S., . . . Li, K. C. (2015). R331W Missense Mutation of Oncogene YAP1 Is a Germline Risk Allele for Lung Adenocarcinoma With Medical Actionability. J Clin Oncol, 33(20), 2303-2310. doi:10.1200/JCO.2014.59.3590

Deng, B., Sun, Z., Jason, W., \& Yang, P. (2013). Increased BCAR1 predicts poor outcomes of non-small cell lung cancer in multiple-center patients. Ann Surg Oncol, 20 Suppl 3, S701-708. doi:10.1245/s10434-013-3184-2

DePristo, M. A., Banks, E., Poplin, R., Garimella, K. V., Maguire, J. R., Hartl, C., . . Daly, M. J. (2011). A framework for variation discovery and genotyping using next-generation DNA sequencing data. Nat Genet, 43(5), 491-498. doi:10.1038/ng.806

Donner, I., Katainen, R., Sipila, L. J., Aavikko, M., Pukkala, E., \& Aaltonen, L. A. (2018). Germline mutations in young non-smoking women with lung adenocarcinoma. Lung Cancer, 122, 76-82. doi:10.1016/j.lungcan.2018.05.027

Erdem, L., Giovannetti, E., Leon, L. G., Honeywell, R., \& Peters, G. J. (2012). Polymorphisms to predict outcome to the tyrosine kinase inhibitors gefitinib, erlotinib, sorafenib and sunitinib. Curr Top Med Chem, 12(15), 1649-1659. doi:10.2174/156802612803531333

Henschke, C. I., Shaham, D., Yankelevitz, D. F., Kramer, A., Kostis, W. J., Reeves, A. P., . . Miettinen, O. S. (2006). CT screening for lung cancer: significance of diagnoses in its baseline cycle. Clin Imaging, 30(1), 11-15. doi:10.1016/j.clinimag.2005.07.003

Huang, W., Deng, B., Wang, R. W., Tan, Q. Y., He, Y., Jiang, Y. G., \& Zhou, J. H. (2012). BCAR1 protein plays important roles in carcinogenesis and predicts poor prognosis in non-small-cell lung cancer. PLoS One, 7(4), e36124. doi:10.1371/journal.pone.0036124

Hung, R. J., McKay, J. D., Gaborieau, V., Boffetta, P., Hashibe, M., Zaridze, D., . . Brennan, P. (2008). A susceptibility locus for lung cancer maps to nicotinic acetylcholine receptor subunit genes on 15q25. Nature, 452(7187), 633-637. doi:10.1038/nature06885

Ikeda, K., Shiraishi, K., Eguchi, A., Osumi, H., Matsuishi, K., Matsubara, E., . . Suzuki, M. (2014). Association of a genetic variant of CYP19A1 with multicentric development of lung adenocarcinomas. Ann Surg Oncol, 21(3), 939-945. doi:10.1245/s10434-013-3362-2

Kanwal, M., Ding, X. J., Ma, Z. H., Li, L. W., Wang, P., Chen, Y., . . Cao, Y. (2018). Characterization of germline mutations in familial lung cancer from the Chinese population. Gene, 641, 94-104. doi:10.1016/j.gene.2017.10.020

Lee, H. Y., Choi, Y. L., Lee, K. S., Han, J., Zo, J. I., Shim, Y. M., \& Moon, J. W. (2014). Pure ground-glass opacity neoplastic lung nodules: histopathology, imaging, and management. AJR Am J Roentgenol, 202(3), W224233. doi:10.2214/AJR.13.11819

Li, H., \& Durbin, R. (2009). Fast and accurate short read alignment with Burrows-Wheeler transform. Bioinformatics, 25(14), 1754-1760. doi:10.1093/bioinformatics/btp324

Liu, Y., Kheradmand, F., Davis, C. F., Scheurer, M. E., Wheeler, D., Tsavachidis, S., . . Spitz, M. R. (2016). Focused Analysis of Exome Sequencing Data for Rare Germline Mutations in Familial and Sporadic Lung Cancer. $J$ Thorac Oncol, 11(1), 52-61. doi:10.1016/j.jtho.2015.09.015

Malhotra, J., Malvezzi, M., Negri, E., La Vecchia, C., \& Boffetta, P. (2016). Risk factors for lung cancer worldwide. Eur Respir J, 48(3), 889-902. doi:10.1183/13993003.00359-2016

Nykamp, K., Anderson, M., Powers, M., Garcia, J., Herrera, B., Ho, Y. Y., . . Topper, S. (2017). Sherloc: a comprehensive refinement of the ACMG-AMP variant classification criteria. Genet Med, 19(10), 11051117. doi:10.1038/gim.2017.37

Raz, D. J., He, B., Rosell, R., \& Jablons, D. M. (2006). Current concepts in bronchioloalveolar carcinoma biology. Clin Cancer Res, 12(12), 3698-3704. doi:10.1158/1078-0432.CCR-06-0457

Renieri, A., Mencarelli, M. A., Cetta, F., Baldassarri, M., Mari, F., Furini, S., . . Frullanti, E. (2014). Oligogenic germline mutations identified in early non-smokers lung adenocarcinoma patients. Lung Cancer, 85(2), 168-174. doi:10.1016/j.lungcan.2014.05.020

Shi, C., Qian, J., Ma, M., Zhang, Y., \& Han, B. (2014). Notch 3 protein, not its gene polymorphism, is associated with the chemotherapy response and prognosis of advanced NSCLC patients. Cell Physiol Biochem, 34(3), 743752. doi:10.1159/000363039

Shukuya, T., Patel, S., Shane-Carson, K., He, K., Bertino, E. M., Shilo, K., . . Carbone, D. P. (2018). Lung Cancer Patients with Germline Mutations Detected by Next-Generation Sequencing and/or Liquid Biopsy. J Thorac Oncol, 13(2), e17-e19. doi:10.1016/j.jtho.2017.09.1962

Peer] reviewing PDF | (2021:01:57035:2:0:NEW 8 Jul 2021) 
275 276
Siegel, R. L., Miller, K. D., \& Jemal, A. (2015). Cancer statistics, 2015. CA Cancer J Clin, 65(1), 5-29. doi:10.3322/caac. 21254

Subramanian, J., \& Govindan, R. (2007). Lung cancer in never smokers: a review. J Clin Oncol, 25(5), 561-570. doi:10.1200/JCO.2006.06.8015

Tomoshige, K., Matsumoto, K., Tsuchiya, T., Oikawa, M., Miyazaki, T., Yamasaki, N., ... Nagayasu, T. (2015). Germline mutations causing familial lung cancer. J Hum Genet, 60(10), 597-603. doi:10.1038/jhg.2015.75

Wang, Y., Wang, C., Zhang, J., Zhu, M., Zhang, X., Li, Z., . . . Shen, H. (2018). Interaction analysis between germline susceptibility loci and somatic alterations in lung cancer. Int J Cancer, 143(4), 878-885. doi:10.1002/ijc.31351

Winther-Larsen, A., Nissen, P. H., Jakobsen, K. R., Demuth, C., Sorensen, B. S., \& Meldgaard, P. (2015). Genetic polymorphism in the epidermal growth factor receptor gene predicts outcome in advanced non-small cell lung cancer patients treated with erlotinib. Lung Cancer, 90(2), 314-320. doi:10.1016/j.lungcan.2015.09.003

Zhang, Y., Zhang, L., Li, R., Chang, D. W., Ye, Y., Minna, J. D., . . . Wu, X. (2017). Genetic variations in cancer-related significantly mutated genes and lung cancer susceptibility. Ann Oncol, 28(7), 1625-1630. doi:10.1093/annonc/mdx161

Zhou, X., Wang, W., Zhang, S., Wang, X., Tang, Z., Gu, J., . . . Huang, J. (2017). CACNA1B (Cav2.2) Overexpression and Its Association with Clinicopathologic Characteristics and Unfavorable Prognosis in Non-Small Cell Lung Cancer. Dis Markers, 2017, 6136401. doi:10.1155/2017/6136401 


\section{Figure 1}

representative of ground glass opacity nodules

Two representative computed tomography images of ground glass opacity nodules. The arrows indicate the nodules.

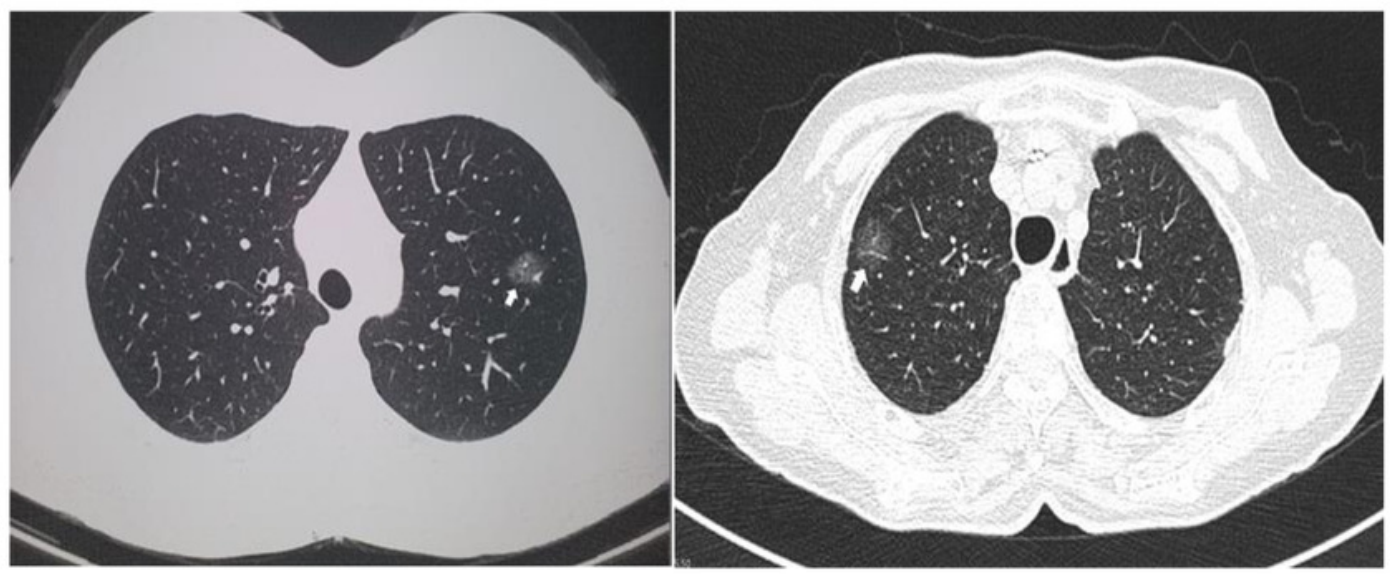


Figure 2

Flowchart of analysis

The stepwise filtering strategy used to screen for potential candidate germline mutations. 
A.

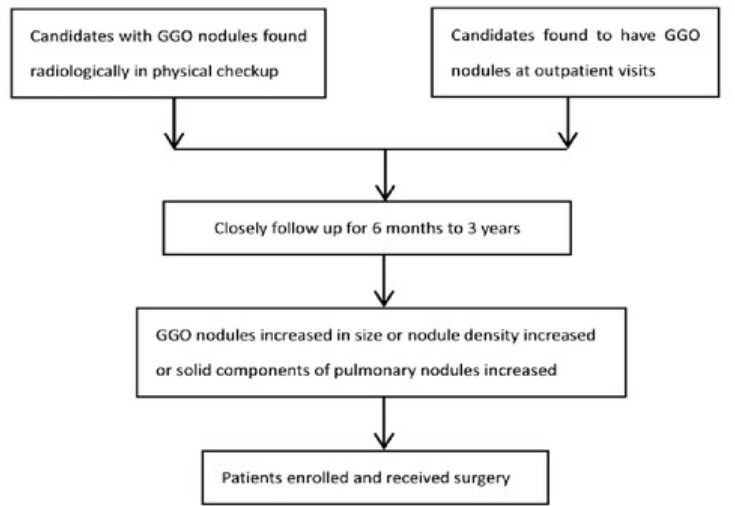

B.

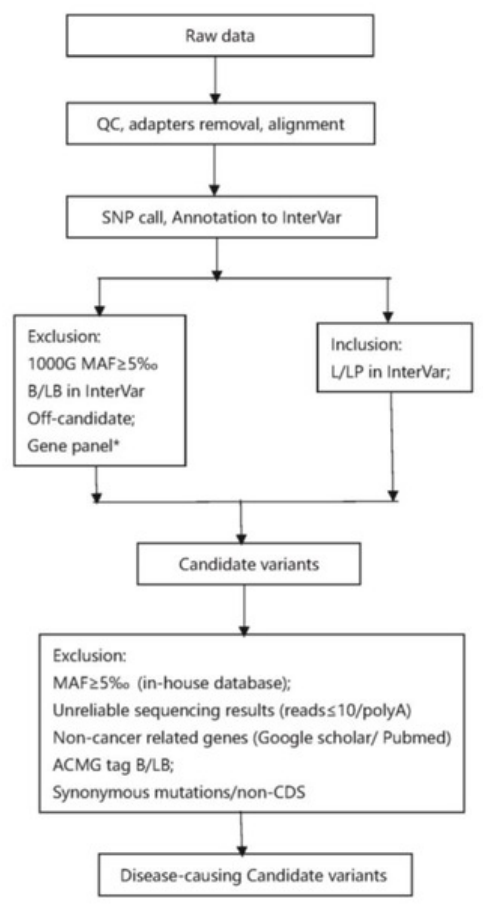


Figure 3

Mutation distriubtion.

The distribution of germline mutations in each patient.

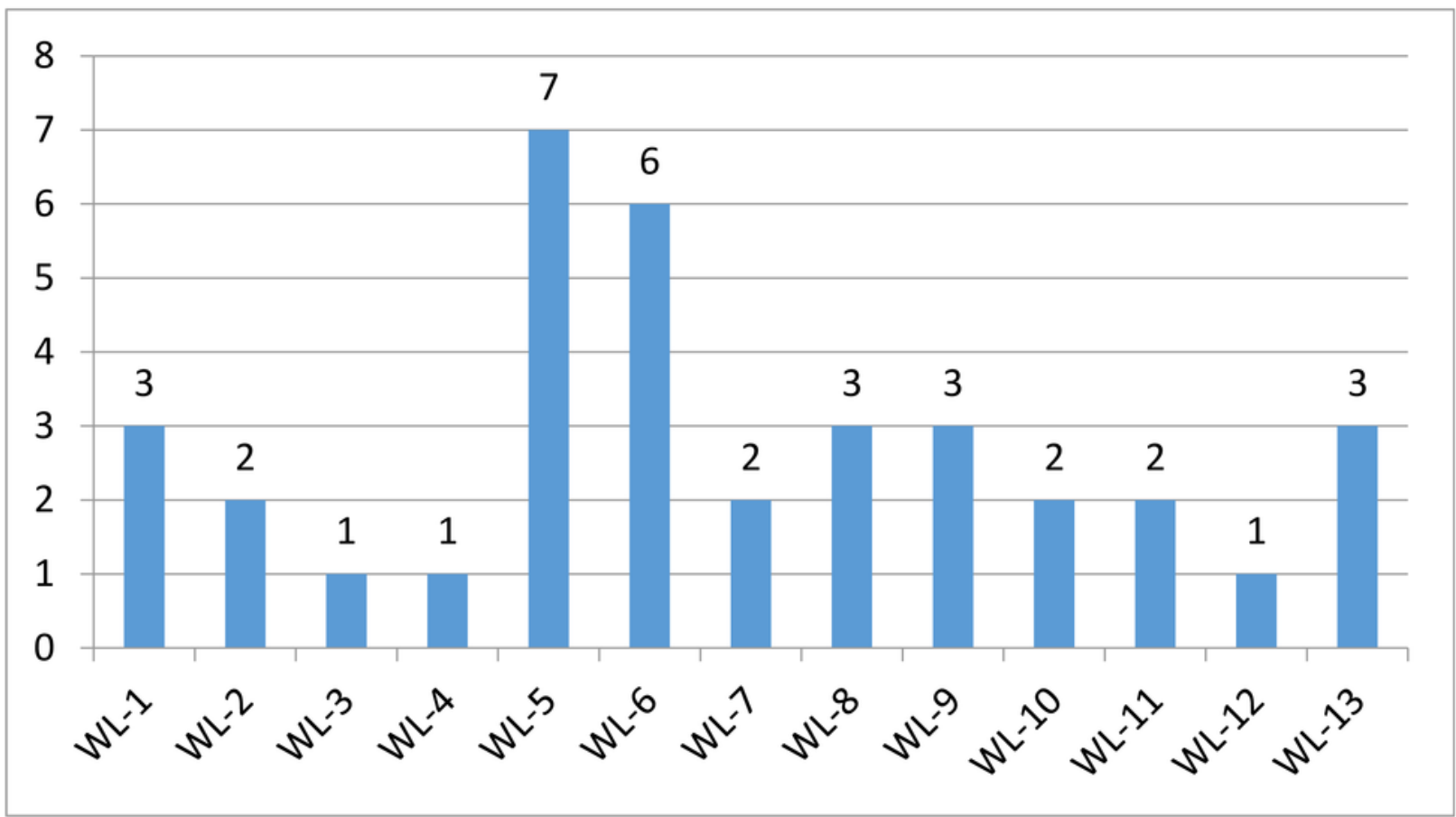




\section{Table 1 (on next page)}

Characteristics of study subjects.

Characteristics of study subjects was collected and analyzed. 
1 Table 1. Characteristics of study subjects.

\begin{tabular}{|c|c|c|}
\hline \multirow{2}{*}{ Characteristics } & & \\
\hline \multirow{2}{*}{ Age at Diagnosis } & Mean (SD) & $61.5(8.7)$ \\
\cline { 2 - 3 } & Range & $48-79$ \\
\hline \multirow{2}{*}{ Gender } & Male (\%) & $2(15.4)$ \\
\cline { 2 - 3 } & Female (\%) & $11(84.6)$ \\
\hline \multirow{2}{*}{ Smoking history } & Non-smokers (\%) & $13(100.0)$ \\
\cline { 2 - 3 } & Smokers (\%) & $0(0)$ \\
\hline
\end{tabular}


Table 2 (on next page)

Clinical information of study subjects.

Pathology, tumor size and tumor location of study objects were shown in details. 
1 Table 2. Clinical information of study subjects.

\begin{tabular}{|c|c|c|c|}
\hline Patient ID & Pathology & $\begin{array}{c}\text { Tumour size } \\
\text { (cm) }\end{array}$ & Tumour location \\
\hline WL-1 & LUAD $\dagger$, invasive & 1.2 & right upper lobe \\
\hline WL-2 & LUAD $\dagger$, AIS* & 0.6 & left upper lobe \\
\hline WL-3 & Atypical adenomatous hyperplasia & 0.5 & right upper lobe \\
\hline WL-4 & LUAD $\dagger$, invasive & 1.5 & left upper lobe \\
\hline WL-5 & LUAD $\dagger$, minimally invasive & 1.0 & right upper lobe \\
\hline WL-6 & LUAD $\dagger$, minimally invasive & 0.6 & left upper lobe \\
\hline WL-7 & LUAD $\uparrow$, invasive & 2.0 & right upper lobe \\
\hline WL-8 & LUAD $\dagger$, invasive & 2.0 & right lower lobe \\
\hline WL-9 & LUAD $\dagger$, AIS* & 0.8 & right upper lobe \\
\hline WL-10 & $\mathrm{LUAD}_{\dagger} \dagger, \mathrm{AIS}^{*}$ & 0.7 & right upper lobe \\
\hline WL-11 & LUAD $\dagger$, AIS* & 0.6 & right lower lobe \\
\hline WL-12 & LUAD $\dagger$, AIS* & 0.8 & right upper lobe \\
\hline WL-13 & LUAD $\dagger$, minimally invasive & 0.7 & right lower lobe \\
\hline
\end{tabular}




\section{Table 3(on next page)}

Summary of potentially deleterious germline mutations in lung cancer cases.

Annotation of potentially deleterious germline mutations in each gene were described in details. 
1 Table 3. Summary of potentially deleterious germline mutations in lung cancer cases.

\begin{tabular}{|c|c|c|c|c|c|c|c|c|c|c|}
\hline Gene & Position & RS & Ref/Alt & Protein alteration & Genetic model & Type of mutation & InterVar annotation & $\begin{array}{l}\text { VAF in } \\
\text { patients }\end{array}$ & VAF in GnomAD_EAS & No. of patients with mutation \\
\hline ANXA1 & Chr 9: 75775752 & & $\mathrm{~A} / \mathrm{T}$ & NM_000700:exon6:C.A418T:p.1140F & - & nonsynonymous SNV & Uncertain significance & 0.0833 & - & 3 \\
\hline Nотснз & Chr 19: 15281611 & - & $\mathrm{T} / \mathrm{G}$ & NM_001 184790:exon8::c.C1070T:p.T357M & $\mathrm{AD}^{*}$ & nonsynonymous SNV & Likely pathogenic & 0.0278 & - & 1 \\
\hline PARD3 & Chr 10: 34671665 & rs1 16642073 & G/A & NM_001 184790:exon8::c.C1070T:p.T357M & & nonsynonymous SNV & Uncertain significance & 0.0833 & 0 & 3 \\
\hline BCAR1 & Chr 16: 75269775 & rs1047683608 & G/A & NM_001170721:exon4:c.C392T:p.P131L & - & nonsynonymous SNV & Uncertain significance & 0.0833 & 0 & 3 \\
\hline CYP21A2 & Chr 6: 32008198 & rs 7755898 & $\mathrm{C} / \mathrm{T}$ & NM_001 128590:exon7:c.C865T:p.Q289X & - & Stopgain & Likely pathogenic & 0.0278 & 0.0001 & 1 \\
\hline LDLRAP1 & Chr 1: 25870253 & - & $\mathrm{G} /-$ & NM_015627:exon1:c.65delG:p.W22fs & $\mathrm{AR}^{* *}$ & frameshift deletion & Pathogenic & 0.0278 & - & 1 \\
\hline CACNA1A & Chr 19: 13319766 & rs373192655 & $\mathrm{C} / \mathrm{T}$ & NM_001 127221:exon46:cGG6587A:p.R2196Q & $\mathrm{AD}$ & nonsynonymous $\mathrm{SNV}$ & Uncertain significance & 0.0556 & 0.0015 & 3 \\
\hline $\operatorname{MAX}$ & Chr 14: 65551007 & rs 756875895 & $\mathrm{G} /-$ & NM_001271068:exon3:c.154delC:p.L52fs & $\mathrm{AD}$ & Frameshift deletion & Likely pathogenic & 0.0278 & - & 2 \\
\hline HSD17B4 & Chr 5: 118814630 & rs7633363391 & $\mathrm{C} / \mathrm{G}$ & NM_001 199292::xon7:c.C482G:p.A161G & $\mathrm{AR}$ & nonsynonymous SNV & Likely pathogenic & 0.0556 & 0.0002 & 2 \\
\hline RAD50 & Chr 5: 131931460 & rs587781454 & $-/ \mathrm{T}$ & NM_005732:exon13:c.2165_2166insT:p.K722fs & - & Frameshift deletion & Uncertain significance & 0.0556 & - & 2 \\
\hline FUS & Chr 16:31195580 & - & $\mathrm{G} / \mathrm{A}$ & NM_001170634:exon5:c.G383A:p.S128N & $\mathrm{AD}$ & nonsynonymous SNV & Uncertain significance & 0.0556 & - & 3 \\
\hline MTAP & Chr 9: 21854717 & rs891972796 & $\mathrm{C} / \mathrm{T}$ & NM_002451:exon6:c.C538T:p.R180W & $\mathrm{AD}$ & nonsynonymous SNV & Uncertain significance & 0.0556 & 0 & 3 \\
\hline TENM4 & Chr 11: 78412907 & - & $\mathrm{T} / \mathrm{C}$ & NM_001098816:exon28:CA4751G:p.Q1584R & $\mathrm{AD}$ & nonsynonymous SNV & Likely pathogenic & 0.0833 & - & 2 \\
\hline GJB2 & Chr 13: 20763485 & rs80338943 & $\mathrm{G} /-$ & NM_004004:exon2:c.235delC:p.L79fs & $\mathrm{AD}$ & Frameshift deletion & Uncertain significance & 0.0556 & - & 2 \\
\hline TTN & Chr 2: 179427779 & rs192360370 & G/A & NM_003319:exon154:c.C55885T:p.R18629C & $\mathrm{AR} / \mathrm{AD}$ & nonsynonymous SNV & Uncertain significance & 0.0278 & 0.0038 & 1 \\
\hline LMO2 & Chr 11: 33886202 & & $\mathrm{C} / \mathrm{G}$ & NM_001142316:exon2:c.G203C:p.G68A & & nonsynonymous SNV & Uncertain significance & 0.0556 & - & 2 \\
\hline
\end{tabular}

2

*autosomal dominant; **autosomal recessive. 